\title{
Chemical Reaction Effects on Free Convective Flow of a Polar Fluid from a Vertical Plate with Uniform Heat and Mass Fluxes
}

\author{
P. M. Patil \\ Department of Mathematics, J S S's Banashankari Arts, Commerce and Shanti Kumar Gubbi Science College, \\ Vidyagiri, Dharwad - 580004, Karnataka, India.
}

\begin{abstract}
This article deals with a study of two dimensional free convective flow of a polar fluid through a porous medium due to combined effects of thermal and mass diffusion in presence of a chemical reaction of first order. The objective of the present investigation is to analyze the free convective flow in the presence of prescribed wall heat flux and mass flux condition. The governing partial differential equations are nondimensionalized and transformed into a system of non-similar equations. The resulting coupled nonlinear partial differential equations are solved numerically under appropriate transformed boundary conditions using an implicit finite difference scheme in combination with quasilinearisation technique. Computations are performed for a wide range of values of the various governing flow parameters of the velocity, angular velocity, temperature and species concentration profiles and results are presented graphically. The numerical results for local skin friction coefficient, couple stress coefficient, local Nusselt number and local Sherwood number are also presented. The obtained results are compared with previously published work and were to be in excellent agreement. The study reveals that the flow characteristics are profoundly influenced by the polar effects.
\end{abstract}

Keywords: Free convection; polar fluid; porous medium; chemical reaction; quasilinearisation.

\section{Introduction}

The flow through a porous medium under the influence of temperature and concentration differences, is one of the most important and contemporary subjects, because it finds great applications in geothermy, geophysics and technology. The practical interest in convective heat and mass transfer through a porous medium has grown rapidly due to the wide range of applications in engineering fields. These important applications include such areas as geothermal energy utilization, thermal energy storage and recoverable systems, petroleum reservoirs, insulation of high temperature gas-solid reaction vessels, chemical catalytic convectors, storage of grain, fruits and vegetables, pollutant dispersion in aquifers, agricultural and water distribution, buried electrical cables, combustion in situ in underground reservoirs for the enhancement of oil recovery, ceramic radiant porous burners used in industrial firms as efficient heat and mass transfer devices and the reduction of hazardous combustion products using catalytic porous beds. An excellent review on this subject can be found in the review article by Cheng [1]. Lee et al. [2] examined the effects of mixed convection along vertical cylinders and needles with uniform surface heat flux using a single curvature parameter as well as single buoyancy parameter to treat the limiting case of natural convection along the surface.

Non-Newtonian fluids in porous media exhibit a non-linear flow behavior that is different from that of Newtonian fluids in porous media. The research on heat and mass transfer in flows of non-Newtonian fluids in porous media is very important due to its practical applications in engineering, such as oil recovery, food processing, the spreading of contaminants in the environment and in various processes in the chemical and materials industry. Chen and Chen [3] presented similarity solutions for natural convection of a non-Newtonian fluid over vertical surfaces in porous media. Nakayama and Koyama [4] studied the natural convection of a nonNewtonian fluid over non-isothermal body of arbitrary shape in a porous medium. Kim and Hyun [5] examined natural convection flow of power-law fluid in an enclosure filled with heat generating porous media. Rastogi and Poulikakos [6] studied double diffusion from a vertical surface embedded in a porous medium saturated with a non-Newtonian fluid. These authors have found that the variation of the wall temperature and concentration necessary to yield a constant heat and mass flux at the wall depended strongly on the power law index. Jumah and Majumdar [7, 8] analyzed heat and mass transfer in free convection flow of non-Newtonian power law fluids with yield stress over a vertical plate in saturated porous media subjected to constant/variable wall temperature and concentration. Recently, Cheng [9] studied heat and mass transfer in natural convection of non-Newtonian power law fluids with yield stress in porous media from a vertical plate with variable wall heat and mass fluxes.

Another important class of fluids, called polar fluids, which is a special family of non-Newtonian fluids, whose constitutive equations were developed by Aero et al. [10] and D'ep [11], exhibits boundary layer phenomenon. In the literature, polar fluids are characterized as those fluids with micro-structures, which are mechanically significant when the characteristic dimension of the problem is of the same order of magnitude as 
the size of the micro-structure. Extensive reviews of the theory can be found in the review article by Cowin [12]. Since the micro-structure size is the same as the average pore size, it is pertinent to study the flow of polar fluids through a porous medium. The examples of fluids which can be modeled as polar fluids are mud, crude oil, body fluids, lubricants with polymer additives, etc. The effects of couple stresses on the flow of polar fluids through a porous medium are studied by Patil and Hiremath [13]. Hiremath and Patil [14] examined the effects of free convection on the oscillatory flow of a couple stress fluids through a porous medium. Effects of MHD on unsteady free convection flow past a vertical porous plate was investigated by Helmy [15]. Steady flow of a polar fluid through a porous medium by using Forchheimer's model was discussed by Raptis and Takhar [16]. Kim [17] analysed the unsteady MHD convection flow of polar fluids past a vertical moving porous plate in a porous medium. Analytical solutions for the problem of the flow of a polar fluid past a vertical porous plate in the presence of couple stresses and radiation, where the temperature of the plate is assumed to oscillate about a mean value are obtained by Ogulu [18]. Patil and Kulkarni [19] examined the effects of chemical reaction on free convective flow of a polar fluid through a porous medium in the presence of internal heat generation. Recently, Patil [20] analysed the effects of free convection on the oscillatory flow of a polar fluid through a porous medium in the presence of variable wall heat flux. Free convective oscillatory flow of a polar fluid through a porous medium in the presence of oscillating suction and temperature was examined by Patil and Kulkarni [21]. Chang and Lee [22] examined the flow and heat transfer characteristics of the free convection on a vertical plate with uniform and constant heat flux in a thermally stratified micropolar fluid.

The study of chemically-reactive flows with heat and mass transfer is also of fundamental interest in engineering science research [23]. Levenspiel [24] provided an excellent discussion of such flows in the context of chemical processes and batch reactor systems. Such flows are also important in cooling tower design, geochemical transport in repositories and the dynamics of fog and mist composition, drying, distribution of temperature and moisture over agricultural fields and groves of fruit trees, damage of crops due to freezing, evaporation at the surface of a water body. Chemical reaction can be modeled as either homogeneous or heterogeneous processes. This depends on whether they occur at an interface or a single phase volume reaction. A homogeneous reaction is one that occurs uniformly throughout a given phase. On the other hand, heterogeneous reaction takes place in a restricted area or within the boundary of a phase. The order of the chemical reaction depends on several factors. One of the simplest chemical reactions is the first-order reaction in which the rate of reaction is directly proportional to the species concentration. Fairbanks and Wilks [25] were among the first researchers to use a homogeneous chemical reaction model in their study of isothermal diffusive flow past a soluble surface. Van Genuchten [26] presented a theoretical model for adsorping and decaying chemically-reactive mass transfer. Das et al. [27] studied the effects of mass transfer on the flow started impulsively past an infinite vertical plate in the presence of wall heat flux and chemical reaction. Muthucumaraswamy and Ganeshan $[28,29]$ studied the impulsive motion of a vertical plate with heat flux/ mass flux/ suction and diffusion of chemically reactive species. Seddeek [30] employed the finite element method for studying the effects of chemical reaction, variable viscosity, thermophoresis, and heat generation/absorption on a boundary layer hydromagnetic flow with heat and mass transfer over a heated surface. Kandasamy et al. [31] examined the effects of chemical reaction on flow, heat and mass transfer with heat source and suction. Kandasamy et al. [32] also examined the chemical reaction effects on Magnetohydrodynamics flow, heat and mass transfer with heat source and suction. Raptis and Perdikis [33] examined the effects of viscous flow over a non-linearly stretching sheet in the presence of chemical reaction and magnetic field.

The aim of the present study is to investigate the effects of chemical reaction on natural convection flow, with heat and mass transfer, of a polar fluid through a porous medium in the presence of couple stresses and uniform wall heat and mass fluxes. The flow configuration is modeled as a vertical plate bounding the porous region filled with fluid containing soluble and insoluble chemical materials. The fluid is modeled as a polar fluid. The system of nonlinear coupled partial differential equations governing the flow is nondimensionalized and transformed into a system of non-similar, coupled nonlinear partial differential equations. The resulting coupled nonlinear equations are solved numerically under appropriate transformed boundary conditions using an implicit finite-difference scheme in combination with a quasi-linearization technique Inouye and Tate [34] and Patil and Roy [35]. The obtained results are compared with some results reported by Lee et al. [2] and Chang and Lee [22] and are found to be in excellent agreement. The study reveals that the flow field is considerably influenced by the combined effects of mass and thermal diffusion in presence of chemical reaction and couple stresses.

\section{Mathematical Formulation}

We consider steady, laminar, two-dimensional natural convection flow of a viscous incompressible polar fluid over a semi-infinite vertical plate embedded in a porous medium. The x-coordinate is measured from the leading edge of the vertical plate and the y-coordinate is measured normal to the plate. The velocity, angular 
velocity, temperature and concentration fields are $(u, v, 0),(0,0, \omega), \mathrm{T}$ and $\mathrm{C}$, respectively. Figure 1 shows the coordinate system and physical model for the flow configuration. The surface is maintained at uniform heat flux $q_{w}$ as well as uniform mass flux $m_{w}$. The concentration of diffusing species is assumed to be very small in comparison with other chemical species far from the surface $C_{\infty}$, and is infinitely small. Hence, the Soret and Dufour effects are neglected. However, the first-order homogeneous chemical reaction is assumed to take place in the flow. All thermo-physical properties of the fluid in the flow model are assumed to be constant except the density variations causing the buoyancy force represented by the body force term in the momentum equation. The Boussinesq approximation is invoked for the fluid properties to relate density changes to temperature and concentration changes, and to couple in this way the temperature and concentration fields to the flow field (Schlichting [36]). Under the above assumptions, the equations of conservation of mass, momentum, angular momentum, energy and concentration governing the free convection boundary layer flow through porous medium are given by (Aero et al. [10], D'ep [11], Patil [20] and Patil and Kulkarni [19, 21]):

$$
\begin{aligned}
& \frac{\partial u}{\partial x}+\frac{\partial v}{\partial y}=0 \\
& u \frac{\partial u}{\partial x}+v \frac{\partial u}{\partial y}=\left(v+v_{r}\right) \frac{\partial^{2} u}{\partial y^{2}}+2 v_{r} \frac{\partial \omega}{\partial y}+g\left[\beta_{T}\left(T-T_{\infty}\right)+\beta_{C}\left(C-C_{\infty}\right)\right]-\left(\frac{v+v_{r}}{K}\right) u \\
& u \frac{\partial \omega}{\partial x}+v \frac{\partial \omega}{\partial y}=\frac{\gamma}{I} \frac{\partial^{2} \omega}{\partial y^{2}} \\
& u \frac{\partial T}{\partial x}+v \frac{\partial T}{\partial y}=\frac{k}{\rho C_{P}} \frac{\partial^{2} T}{\partial y^{2}} \\
& u \frac{\partial C}{\partial x}+v \frac{\partial C}{\partial y}=D \frac{\partial^{2} C}{\partial y^{2}}-k_{1}\left(C-C_{\infty}\right),
\end{aligned}
$$

where $\gamma=\left(C_{a}+C_{d}\right) I^{-1}$. All of the parameters appearing in Eqs. (1)- (5) are defined in the Nomenclature section.

The appropriate boundary conditions are:

$$
\begin{aligned}
& y=0 \quad: \quad u=0, \quad v=0, \quad \frac{\partial \omega}{\partial y}=-m \frac{\partial^{2} u}{\partial y^{2}}, \quad-k \frac{\partial T}{\partial y}=q_{w}, \quad-D \frac{\partial C}{\partial y}=m_{w}, \\
& y \rightarrow \infty: \quad u \rightarrow 0, \quad \omega \rightarrow 0, \quad T \rightarrow T_{\infty}, \quad C \rightarrow C_{\infty} \text {. }
\end{aligned}
$$

The boundary conditions (6) are derived on the basis of the assumption that the couple stresses are dominant during the rotation of the particles. Further, $m$ is a constant and $0 \leq m \leq 1$. The case $m=0$, which corresponds to $\frac{\partial \omega}{\partial y}=0$ at the wall represents concentrated particle flows in which the micro elements close to the wall surface are not able to rotate. This case is known as strong concentration of micro elements. The case $m=0.5$, which corresponds to the vanishing of antisymmetric part of the stress tensor and indicates weak concentration of micro elements. The case $m=1.0$, which corresponds to the modeling of turbulent boundary layer flows. Here, we shall consider the case of $m=0.5$. 


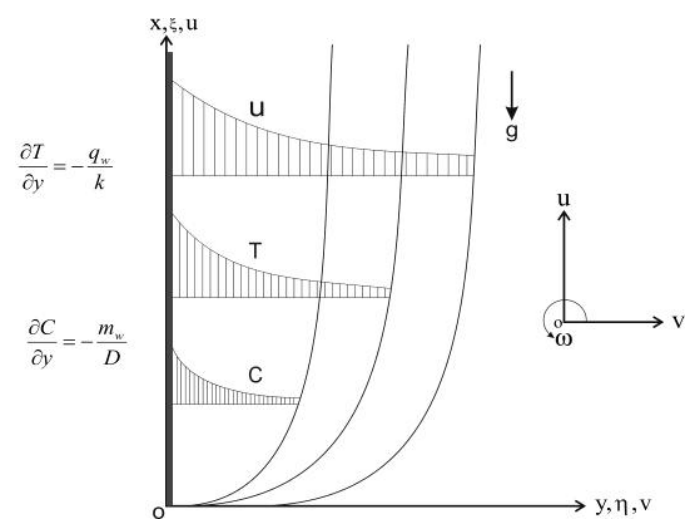

Fig. 1. Flow model and coordinate system

Let $\psi(x, y)$ represent the stream function, then

$u=\frac{\partial \psi}{\partial y}, v=-\frac{\partial \psi}{\partial x}$.

Substituting the following transformations:

$$
\begin{aligned}
& \xi=\frac{x}{L}, \eta=\frac{G r^{1 / 5}}{L \xi^{1 / 5}} y, \psi(x, y)=v G r^{1 / 5} \xi^{4 / 5} f(\xi, \eta), \Omega(\xi, \eta)=\frac{L^{2}}{v G r^{3 / 5} \xi^{2 / 5}} \omega(x, y), \\
& \Theta(\xi, \eta)=\frac{\left(T-T_{\infty}\right)}{\left(q_{w} / k\right) L \xi^{1 / 5}} G r^{1 / 5}, \Phi(\xi, \eta)=\frac{\left(C-C_{\infty}\right)}{\left(m_{w} / D\right) L \xi^{1 / 5}} G r^{1 / 5}, u=\frac{\partial \psi}{\partial y}=\frac{v}{L} G r^{2 / 5} \xi^{3 / 5} \frac{\partial f}{\partial \eta}, \\
& v=-\frac{\partial \psi}{\partial x}=-\frac{v}{L} G r^{1 / 5} \xi^{4 / 5}\left\{\frac{4}{5} \frac{f}{\xi}+\frac{\partial f}{\partial \xi}+L \frac{\partial f}{\partial \eta} \eta_{x}\right\}, G r=\frac{g \beta_{T} q_{w} L^{4}}{k v^{2}}, G r^{*}=\frac{g \beta_{C} m_{w} L^{4}}{D v^{2}}, \lambda=\frac{G r^{*}}{G r}, \\
& D a=\frac{K}{G r^{2 / 5} L^{2}}, \operatorname{Pr}=\frac{\rho v C_{P}}{k}, \alpha=\frac{v_{r}}{v}, \beta=\frac{I v}{\gamma}, S c=\frac{v}{D}, \Delta=\frac{k_{1} L^{2}}{v G r^{2 / 5}},
\end{aligned}
$$

into Eqs. (2) - (5), we obtain the following non-dimensional equations:

$$
\begin{aligned}
(1+\alpha) \frac{\partial^{3} f}{\partial \eta^{3}}+\frac{4}{5} f \frac{\partial^{2} f}{\partial \eta^{2}}-\frac{3}{5}\left(\frac{\partial f}{\partial \eta}\right)^{2}+2 \alpha \frac{\partial \Omega}{\partial \eta}+\Theta+ & \lambda \Phi-\frac{(1+\alpha)}{D a} \xi^{2 / 5} \frac{\partial f}{\partial \eta} \\
& =\xi\left\{\frac{\partial f}{\partial \eta} \frac{\partial^{2} f}{\partial \xi \partial \eta}-\frac{\partial f}{\partial \xi} \frac{\partial^{2} f}{\partial \eta^{2}}\right\}
\end{aligned}
$$

where Eq. (7) implies that the continuity equation is identically satisfied.

The corresponding boundary conditions (6) reduce to the following non-dimensional form: 


$$
\begin{aligned}
& \frac{\partial f}{\partial \eta}=0, \frac{\partial \Omega}{\partial \eta}=-\frac{1}{2} \frac{\partial^{3} f}{\partial \eta^{3}}, \frac{\partial \Theta}{\partial \eta}=-1, \frac{\partial \Phi}{\partial \eta}=-1 \text { at } \eta=0, \\
& \frac{\partial f}{\partial \eta} \rightarrow 0, \Omega \rightarrow 0, \Theta \rightarrow 0, \Phi \rightarrow 0 \text { as } \eta \rightarrow \infty .
\end{aligned}
$$

The quantities of physical interest, namely, the skin-friction coefficient, wall couple stress coefficient, local Nusselt number and the local Sherwood number are defined, respectively, as

$$
\begin{aligned}
& C_{f x}=\frac{2\left(\mu+\mu_{r}\right)}{\rho U^{2}} \frac{\partial u}{\partial y}=2(1+\alpha) G r^{11 / 25} \xi^{1 / 5} f_{\eta \eta}(\xi, 0), \\
& \text { i.e. } C_{f x} G r^{-11 / 25}=2(1+\alpha) \xi^{1 / 5} f_{\eta \eta}(\xi, 0) . \\
& C_{m f}=\frac{\gamma}{I \rho U L} \frac{\partial \omega}{\partial y}=\frac{B}{\beta} G r^{2 / 5} \xi^{1 / 5} \Omega_{\eta}(\xi, 0), \\
& \text { i.e. } C_{m f} G r^{-2 / 5}=\frac{B}{\beta} \xi^{1 / 5} \Omega_{\eta}(\xi, 0) . \\
& N u_{x}=\frac{h L}{k}, \\
& \text { i.e. } N u_{x} G r^{-1 / 5}=\frac{1}{\Theta(\xi, 0)} .
\end{aligned}
$$

and

$$
\begin{aligned}
& S h_{x}=\frac{h m L}{D}, \\
& \text { i.e. } S h_{x} G r^{-1 / 5}=\frac{1}{\Phi(\xi, 0)} .
\end{aligned}
$$

Where $U=\frac{v G r^{2 / 5} \xi^{3 / 5}}{L}$ and $B=\frac{v}{\rho L^{2}}$.

\section{Numerical Procedure}

The set of non-dimensional equations (9)-(12) under the boundary conditions (13) for uniform wall heat flux as well as uniform mass flux with the initial conditions obtained from the corresponding steady state equations have been solved numerically using an implicit finite-difference scheme in combination with the quasi-linearization technique by Inouye and Tate [34] and Patil and Roy [35].

Denoting, $f_{\eta}(\xi, \eta)=F(\xi, \eta)$, where $f(\xi, \eta)=\int_{0}^{\eta} F(\xi, \eta) d \eta$,

then Eqs. (9) - (12) take the form:

$$
\begin{aligned}
& F_{\eta \eta}+\frac{4}{5(1+\alpha)} f F_{\eta}-\frac{3}{5(1+\alpha)} F^{2}+\frac{2 \alpha}{(1+\alpha)} \Omega_{\eta}+\frac{1}{(1+\alpha)}(\Theta+\lambda \Phi)-\frac{\xi^{2 / 5}}{D a} F= \\
& \frac{\xi}{(1+\alpha)}\left[F F_{\xi}-f_{\xi} F_{\eta}\right], \\
& \Omega_{\eta \eta}+\frac{4}{5} \beta f \Omega_{\eta}-\frac{2}{5} \beta F \Omega=\beta \xi\left[F \Omega_{\xi}-f_{\xi} \Omega_{\eta}\right], \\
& \Theta_{\eta \eta}+\frac{4}{5} \operatorname{Pr} f \Theta_{\eta}-\frac{1}{5} \operatorname{Pr} F \Theta=\operatorname{Pr} \xi\left[F \Theta_{\xi}-f_{\xi} \Theta_{\eta}\right], \\
& \Phi_{\eta \eta}+\frac{4}{5} S c f \Phi_{\eta}-\frac{1}{5} S c F \Phi-S c \Delta \xi^{2 / 5} \Phi=S c \xi\left[F \Phi_{\xi}-f_{\xi} \Phi_{\eta}\right],
\end{aligned}
$$

and the boundary conditions (13) reduce to: 
$\eta=0: F=0, \Omega_{\eta}=-\frac{1}{2} F_{\eta \eta}, \Theta_{\eta}=-1, \quad \Phi_{\eta}=-1$,

$\eta \rightarrow \infty: F \rightarrow 0, \quad \Omega \rightarrow 0, \quad \Theta \rightarrow 0, \quad \Phi \rightarrow 0$.

An iterative sequence of linear equations is carefully constructed to approximate the nonlinear equations (19)-(22) under the boundary conditions (23) achieving quadratic convergence and monotonicity. Applying the quasi-linearization technique, the nonlinear coupled ordinary differential equations (19)-(22) with boundary conditions (23) yield the following sequence of linear ordinary differential equations:

$$
\begin{aligned}
& F_{\eta \eta}{ }^{(i+1)}+A_{1}{ }^{i} F_{\eta}{ }^{(i+1)}+A_{2}^{i} F^{(i+1)}+A_{3}^{i} \Omega_{\eta}{ }^{(i+1)}+A_{4}{ }^{i} \Omega_{\eta}{ }^{(i+1)}+A_{5}{ }^{i} \Theta^{(i+1)}+A_{6}{ }^{i} \Phi^{(i+1)}=A_{7}{ }^{i}, \\
& \Omega_{\eta \eta}{ }^{(i+1)}+B_{1}{ }^{i} \Omega_{\eta}{ }^{(i+1)}+B_{2}{ }^{i} \Omega^{(i+1)}+B_{3}{ }^{i} \Omega_{\xi}{ }^{(i+1)}+B_{4}{ }^{i} F^{(i+1)}=B_{5}{ }^{i} \text {, } \\
& \Theta_{\eta \eta}{ }^{(i+1)}+C_{1}{ }^{i} \Theta_{\eta}{ }^{(i+1)}+C_{2}{ }^{i} \Theta^{(i+1)}+C_{3}{ }^{i} \Theta_{\xi}{ }^{(i+1)}+C_{4}{ }^{i} F^{(i+1)}=C_{5}{ }^{i}, \\
& \Phi_{\eta \eta}{ }^{(i+1)}+D_{1}^{i} \Phi_{\eta}{ }^{(i+1)}+D_{2}^{i} \Phi^{(i+1)} D_{3}{ }^{i} \Phi_{\xi}{ }^{(i+1)}+D_{4}{ }^{i} F^{(i+1)}=D_{5}{ }^{i} \text {, } \\
& \text { where, } A_{1}^{i}=\frac{1}{(1+\alpha)}\left[\frac{4}{5} f+\xi f_{\xi}\right], A_{2}^{i}=-\left[\frac{6 F}{5(1+\alpha)}+\frac{\xi}{(1+\alpha)} F_{\xi}+\frac{\xi^{2 / 5}}{D a}\right], A_{3}^{i}=-\frac{\xi}{(1+\alpha)} F \text {, } \\
& A_{4}{ }^{i}=\frac{2 \alpha}{(1+\alpha)}, A_{5}{ }^{i}=\frac{1}{(1+\alpha)}, A_{6}{ }^{i}=\frac{\lambda}{(1+\alpha)}, \quad A_{7}{ }^{i}=-\frac{1}{(1+\alpha)}\left[\frac{3}{5} F+\xi F_{\xi}\right], \quad B_{1}^{i}=\beta\left[\frac{4}{5} f+\xi f_{\xi}\right], \\
& B_{2}{ }^{i}=-\frac{2}{5} \beta F, B_{3}{ }^{i}=-\xi \beta F, B_{4}{ }^{i}=-\beta\left[\frac{2}{5} \Omega+\xi \Omega_{\xi}\right], B_{5}{ }^{i}=B_{4}{ }^{i} F, C_{1}{ }^{i}=\operatorname{Pr}\left[\frac{4}{5} f+\xi f_{\xi}\right] \text {, } \\
& C_{2}{ }^{i}=-\frac{1}{5} \operatorname{Pr} F, C_{3}{ }^{i}=-\xi \operatorname{Pr} F, C_{4}{ }^{i}=-\left[\frac{1}{5} \Theta+\xi \Theta_{\xi}\right] \operatorname{Pr}, C_{5}{ }^{i}=-\left[\frac{1}{5} \Theta+\xi \Theta_{\xi}\right] \operatorname{Pr} F, \\
& D_{1}{ }^{i}=S c\left[\frac{4}{5} f+\xi f_{\xi}\right], D_{2}{ }^{i}=-S c\left[\frac{F}{5}+\Delta \xi^{2 / 5}\right], D_{3}{ }^{i}=-S c \xi F, D_{4}{ }^{i}=-S c\left[\frac{\Phi}{5}+\xi \Phi_{\xi}\right], \\
& D_{5}{ }^{i}=-S c\left[\frac{\Phi}{5}+\xi \Phi_{\xi}\right] F \text {. }
\end{aligned}
$$

The coefficient functions with iterative index $i$ are known and the functions with iterative index $(i+1)$ are to be determined. The boundary conditions are given by

$$
\begin{aligned}
& F^{(i+1)}=0, \quad \Omega_{\eta}{ }^{(i+1)}=-\frac{1}{2} F_{\eta \eta}{ }^{(i+1)}, \quad \Theta_{\eta}{ }^{(i+1)}=\Phi_{\eta}{ }^{(i+1)}=-1 \quad \text { at } \eta=0, \\
& F^{(i+1)} \rightarrow 0, \quad \Omega^{(i+1)} \rightarrow 0, \quad \Theta^{(i+1)}=\Phi^{(i+1)} \rightarrow 0, \quad \text { as } \eta \rightarrow \eta_{\infty} .
\end{aligned}
$$

where $\eta_{\infty}$ is the edge of the boundary layer.

Since the method is explained by Inouye and Tate [34] and also in a recent paper by Patil and Roy [35], its detailed analysis is not presented here for the sake of brevity. In brief, the nonlinear coupled ordinary differential equations were replaced by an iterative sequence of linear equations following quasi-linearization technique. The resulting sequences of linear ordinary differential equations were expressed in difference form using central difference scheme in $\eta$ - direction. In each iteration step, the equations were then reduced to a system of linear algebraic equations with a block tri-diagonal structure which is solved by using Varga algorithm [37].

To ensure the convergence of the numerical solution to the exact physical solution, the step size $\Delta \eta$ and the edge of the boundary layer have been optimized and the results presented here are independent of the step size at least up to the fifth decimal place. The step size of $\Delta \eta$ has been taken as 0.01 . A convergence criterion based on the relative difference between the current and previous iteration values is employed. When the value of this difference reaches less than $10^{-5}$, the solution is assumed to have converged and the iterative process is terminated. Accuracy of the presented approach is verified by direct comparison with the results previously reported by Lee et al. [2] and Chang and Lee [22] for a free convective flow of a Newtonian fluid 
along a vertical flat plate $(\alpha=0, \Delta=0, \lambda=0$ and $1 / \mathrm{Da}=0)$. The results of this comparison are presented in Table 1 and are found to be in excellent agreement.

\section{Results and Discussion}

The numerical computations have been carried out for various values of the parameters, namely, $\alpha(1.0 \leq \alpha \leq 3.0), \quad \beta(2.0 \leq \beta \leq 9.0), \quad \lambda(0.0 \leq \lambda \leq 5.0), \quad S c(0.22 \leq S c \leq 2.64)$, $\Delta(-0.5 \leq \Delta \leq 1.0), \quad \operatorname{Pr}(0.01 \leq \operatorname{Pr} \leq 100)$ and $\quad D a(1.0 \leq D a \leq 100000000.0)$. The edge of the boundary layer $\eta_{\infty}$ is taken between 2.5 and 25.0 depending on the values of the parameters.

The effects of ratio of buoyancy forces parameter $(\lambda)$ and Prandtl number $(\operatorname{Pr})$ on the velocity $F(\xi, \eta)$, angular velocity $\Omega(\xi, \eta)$, temperature $\Theta(\xi, \eta)$ and concentration $\Phi(\xi, \eta)$ are presented in Figs. 2-5. The velocity profiles $F(\xi, \eta)$ and skin-friction coefficient $\left(C_{f x} G r^{-11 / 25}\right)$ results are displayed in Figs. 2a and 2b, respectively, for different values buoyancy ratio parameter $(\lambda)$ and Prandtl number (Pr). For aiding flow $(\lambda>0)$, the ratio of buoyancy forces shows the significant overshoot in the velocity profiles near the surface for lower and higher Prandtl numbers $(\operatorname{Pr}=100)$ fluid. Although, the magnitude of the overshoot increases with the ratio of buoyancy forces parameter $\lambda(\lambda>0)$, it decreases as the Prandtl number $(P r)$ increases. The physical reason is that the buoyancy force $(\lambda)$ affects more in smaller Prandtl number fluid $(\operatorname{Pr}=0.01)$ due to the lower viscosity of the fluid. Hence, the velocity increases within the boundary layer due to combined effects of assisting buoyancy forces, acts like a favorable pressure gradient and the velocity overshoot occurs. For higher Prandtl numbers $(P r=100)$ fluids the overshoot is less present as compared to the lower Prandtl numbers $(P r=0.01)$. Because a higher Prandtl number $(P r)$ with more viscous fluid which makes it less sensitive to the buoyancy forces. The effects of $\lambda$ (the ratio of buoyancy forces) and Prandtl number $(P r)$ on the skin-friction coefficient $\left(G r^{-11 / 25} C_{f x}\right)$ when $\alpha=1.0, \beta=4.0, S c=0.22, \Delta=0.5, \xi=1.0$ and $D a=1.0$ are shown in Fig.2b. Results indicate that the skin-friction coefficient $\left(G r^{-11 / 25} C_{f x}\right)$ increases with buoyancy force for both lower and higher Prandtl numbers monotonously. This is due to the fact that the increase of $\lambda$ enhances the fluid acceleration and hence, the skin-friction coefficient increases. However, skin-friction coefficient $\left(G r^{-11 / 25} C_{f x}\right)$ increases for lower Prandtl number fluid. In particular for $\operatorname{Pr}=100$ at $\xi=1.0$, the skin-friction coefficient $\left(G r^{-11 / 25} C_{f x}\right)$ increases approximately $127 \%$ as $\lambda$ increases from $\lambda=1.0$ to 3.0 while for $\operatorname{Pr}=0.01$ the skin-friction coefficient $\left(G r^{-11 / 25} C_{f x}\right)$ increases approximately about $18 \%$ when $\lambda$ increases from $\lambda=1.0$ to 3.0 .

In Figs. 3a and $3 b$, the angular velocity profiles $\Omega(\xi, \eta)$ and wall couple stress coefficient $\left(G r^{-2 / 5} C_{m f}\right)$ results for different values of buoyancy parameter $(\lambda)$ and Prandtl number $(\operatorname{Pr})$ are displayed, respectively. The negative values of the dimensionless angular velocity indicate that the micro rotation of substructures in the polar fluid is in the clock-wise direction. It is observed that the variation of $\lambda$ leads to decrease in the angular velocity. Further, the angular velocity is found to increase with the higher Prandtl number $(P r)$ near the surface due to polar effects. The wall couple stress coefficient $\left(G r^{-2 / 5} C_{m f}\right)$ increases with buoyancy for both lower and higher Prandtl number $(P r)$ fluid monotonously as can be seen in Fig. 3b. In particular at $\xi=1.0,\left(G r^{-2 / 5} C_{m f}\right)$ increases approximately $28 \%$ as $\lambda$ increases from $\lambda=1.0$ to 3.0 when $\mathrm{Pr}$ $=0.01$ while for $\operatorname{Pr}=100,\left(G r^{-2 / 5} C_{m f}\right)$ increases approximately $137 \%$ when $\lambda$ increases from $\lambda=1.0$ to 3.0 .

The temperature $\Theta(\xi, \eta)$ and heat transfer coefficient $\left(G r^{-1 / 5} N u_{x}\right)$ profiles are plotted in Figs. 4a and $4 \mathrm{~b}$, respectively, for different values buoyancy parameter $(\lambda)$ and Prandtl number $(\operatorname{Pr})$. The results indicate that an increase in the buoyancy parameter $(\lambda)$ as well as the Prandtl number $(P r)$ clearly induces a strong reduction 
in the temperature of the fluid and thus resulting in a thinner thermal boundary layer. It is further observed that the heat transfer coefficient $\left(G r^{-1 / 5} N u_{x}\right)$ increases with $\lambda$ for both higher and lower Prandtl number $(P r)$ consistently. It is also observed that for higher Prandtl number $(P r)$, the heat transfer coefficient $\left(G r^{-1 / 5} N u_{x}\right)$ is found to reduce considerably compared to the lower Prandtl number fluid. In particular, at $\xi=$ 1.0, $\left(G r^{-1 / 5} N u_{x}\right)$ increases approximately just about $3 \%$ as $\lambda$ increases from $\lambda=1.0$ to 3.0 with $\operatorname{Pr}=0.01$ while for $\operatorname{Pr}=100,\left(G r^{-1 / 5} N u_{x}\right)$ increases approximately about $16 \%$ when $\lambda$ increases from $\lambda=1.0$ to 3.0 .

In Figs. $5 \mathrm{a}$ and $5 \mathrm{~b}$, the concentration $\Phi(\xi, \eta)$ and mass transfer coefficient $\left(G r^{-1 / 5} S h_{x}\right)$ profiles for different values of the buoyancy ratio parameter $(\lambda)$ and Prandtl number $(P r)$ are depicted, respectively. It is seen that the concentration profile decreases with increasing values of $\lambda$. However, the influence of buoyancy on the concentration profile is very less for both higher and lower Prandtl number. The behavior of the mass transfer rate $\left(G r^{-1 / 5} S h_{x}\right)$ with $\lambda$ is opposite to the case of heat transfer coefficient $\left(G r^{-1 / 5} S h_{x}\right)$. In particular at $\xi=1.0,\left(G r^{-1 / 5} S h_{x}\right)$ increases approximately within $5 \%$ as $\lambda$ increases from $\lambda=1.0$ to 3.0 with $\operatorname{Pr}=0.01$ while for $\operatorname{Pr}=100$, there is no impact of buoyancy on $\left(G r^{-1 / 5} S h_{x}\right)$ as $\lambda$ increases from $\lambda=1.0$ to 3.0.

The effects of the Darcy number $(D a)$ and the material parameter $(\alpha)$ on the velocity $F(\xi, \eta)$, angular velocity $\Omega(\xi, \eta)$, temperature $\Theta(\xi, \eta)$ and the concentration $\Phi(\xi, \eta)$ profiles are displayed in Figs. 6-9. The velocity $F(\xi, \eta)$ and the skin-friction coefficient $\left(G r^{-11 / 25} C_{f x}\right)$ profiles are presented for different values Darcy number $(D a)$ and the material parameter $(\alpha)$ in Figs. 6a and 6b, respectively. It is observed from Fig. 6 a that the velocity $F(\xi, \eta)$ profile decreases considerably with increasing values of the material parameter $(\alpha)$. The significant overshoot near the surface in the velocity profile is observed for the case without the porous medium $(D a \rightarrow \infty)$. However, it is observed that the velocity decreases in the presence of the porous medium $(D a=1.0)$ as compared to the case without the porous medium $(D a \rightarrow \infty)$. The effects of the Darcy number $(D a)$ and the material parameter $(\alpha)$ on the skin-friction coefficient $\left(G r^{-11 / 25} C_{f x}\right)$ when $\lambda=1.0, \beta=4.0, S c=0.22, \Delta=0.5, P r=0.7$ and $\xi=1.0$ are shown in Fig. 6b. It is observed that the skin-friction coefficient $\left(G r^{-11 / 25} C_{f x}\right)$ increases considerably with increasing values of the material parameter $(\alpha)$. This is due to the fact that the increase of $(\alpha)$ enhances the fluid acceleration and hence, the skin-friction coefficient increases. In particular for $\alpha=1.0$ at $\xi=1.0$, the skin-friction coefficient increases approximately about $24 \%$ as $\alpha$ increases from $\alpha=1.0$ to 3.0 with a porous medium $(D a=1.0)$ while for the case without a porous medium $(D a \rightarrow \infty)$, the skin-friction coefficient increases approximately $35 \%$ when $\alpha$ increases from $\alpha=1.0$ to 3.0 .

The angular velocity $\Omega(\xi, \eta)$ and the wall couple stress coefficient $\left(G r^{-2 / 5} C_{m f}\right)$ profiles are plotted in Figs. 7a and 7b, respectively, for different values of Darcy number $(D a)$ and material parameter $(\alpha)$. It is noted that the variation of $(\alpha)$ leads to a considerable increase in the magnitude of the angular velocity. Further, the angular velocity is found to decrease in the presence of the porous medium $(D a=1.0)$ near the surface and away from the surface increases. However, the angular velocity $\Omega(\xi, \eta)$ is found to be reduced when the porous medium is present in comparison to the case without the 
porous medium. The wall couple stress coefficient $\left(G r^{-2 / 5} C_{m f}\right)$ increases with $\alpha$ monotonously for increasing $\xi$ in both the cases with or without the porous medium. In particular at $\xi=1.0$, $\left(G r^{-2 / 5} C_{m f}\right)$ decreases approximately about $42 \%$ as $\alpha$ increases from 1.0 to 3.0 when the porous medium is present while for the case without the porous medium, the wall couple stress coefficient decreases approximately about $45 \%$ when $\alpha$ increases from $\alpha=1.0$ to 3.0 .

The effects of the Darcy number $(D a)$ and the material parameter $(\alpha)$ on the temperature $\Theta(\xi, \eta)$ and the heat transfer rate $\left(G r^{-1 / 5} N u_{x}\right)$ profiles are shown in Figs. $8 \mathrm{a}$ and $8 \mathrm{~b}$. These results indicate that an increase in the material parameter $(\alpha)$ clearly induces a strong increase in the temperature of the fluid and thus results in a thicker thermal boundary layer while in the absence of the porous medium $(D a \rightarrow \infty)$, the temperature profile induces a reduction in the fluid temperature and hence yielding a thinner thermal boundary layer. It is further observed that the heat transfer coefficient $\left(G r^{-1 / 5} N u_{x}\right)$ decreases with variation of the material parameter $(\alpha)$. In particular at $\xi=1.0,\left(G r^{-1 / 5} N u_{x}\right)$ decreases approximately about $16 \%$ as the material parameter $(\alpha)$ increases from 1.0 to 3.0 in the presence of the porous medium $(D a=1.0)$ while for the case without the porous medium $(D a \rightarrow \infty),\left(G r^{-1 / 5} N u_{x}\right)$ decreases approximately $10 \%$ when the material parameter $(\alpha)$ increases from 1.0 to 3.0 .

Figures 9a and 9b illustrate the influence of the Darcy number $(D a)$ and the material parameter $(\alpha)$ on the concentration $\Phi(\xi, \eta)$ and the mass transfer rate $\left(G r^{-1 / 5} S h_{x}\right)$, respectively. It is noted that an increase in the value of the material parameter $(\alpha)$ leads to a rise in the concentration profile while it falls for the case when the porous medium is absent $(D a \rightarrow \infty)$. It is further observed that the mass transfer coefficient decreases with the material parameter $(\alpha)$ for an increasing $\xi$ monotonously. In particular at $\xi=1.0$, $\left(G r^{-1 / 5} S h_{x}\right)$ increases approximately about $7 \%$ as the material parameter $(\alpha)$ increases from 1.0 to 3.0 for $(D a=1.0)$ while for $(D a \rightarrow \infty),\left(G r^{-1 / 5} S h_{x}\right)$ increases approximately about $4 \%$ when the material parameter $(\alpha)$ increases from 1.0 to 3.0 .

The effects of the material parameters $(\alpha)$ and $(\beta)$ on the angular velocity $\Omega(\xi, \eta)$ and the wall couple stress coefficient $\left(G r^{-2 / 5} C_{m f}\right)$ profiles are displayed in Figs. 10a and 10b, respectively. It is noted that the angular velocity profile increases with increasing values of the material parameters $(\alpha)$ and $(\beta)$. It clearly indicates that the wall couple stresses are dominant during the rotation of the particles. It is further noted that the wall couple stress coefficient $\left(G r^{-2 / 5} C_{m f}\right)$ decreases significantly with increasing values of the material parameters $(\alpha)$ and $(\beta)$. In particular at $\xi=1.0,\left(G r^{-2 / 5} C_{m f}\right)$ decreases approximately about $78 \%$ as $(\beta)$ increases from 2.0 to 9.0 at $\alpha=1.0$ and $\alpha=3.0$, respectively.

Figures $11 \mathrm{a}$ and $12 \mathrm{a}$ are displayed the variations of the velocity $F(\xi, \eta)$ and concentration $\Phi(\xi, \eta)$ profiles for various values of the Schmidt number $S c$ and the chemical reaction parameter $\Delta$, respectively. It is observed that the magnitude of the velocity and concentration distributions increase significantly, when the chemical reaction parameter $\Delta<0$ (species consumption or destructive chemical reaction), is increased. An increase in the concentration of the diffusing species increases the mass diffusion and thus, in turn, the fluid velocity increases. On the contrary, for $\Delta>0$ (species generation or constructive chemical reaction), as $\Delta$ increases the velocity distribution decreases, so that the concentration reduces. The values of the Schmidt 
number $(S c)$ are chosen to be more realistic, $0.22,0.66,2.64$, representing diffusing chemical species of most common interest like water, Propyl Benzene hydrogen, water vapor and Propyl Benzene, etc., at 25 degrees Celsius at one atmospheric pressure. It is also observed that the concentration and velocity boundary layers are decreased as the Schmidt number $S c$ is increased. The physical reason is that an increase in the value of the Schmidt number $S c$ leads to a thinning of the concentration boundary layer. As a result, the concentration of the fluid decreases and this leads to a decrease in the fluid velocity.

The variations of the skin-friction coefficient $\left(G r^{-11 / 25} C_{f x}\right)$ and the mass transfer rate $\left(G r^{-1 / 5} S h_{x}\right)$ are presented in Figs. $11 \mathrm{~b}$ and $12 \mathrm{~b}$, respectively. The skin-friction coefficient decreases for increasing values of the chemical reaction parameter $\Delta$ while the mass transfer rate increases for increasing values of the chemical reaction parameter $\Delta$. In particular at $\xi=1.0,\left(G r^{-11 / 25} C_{f x}\right)$ decreases approximately about $22 \%$ as the chemical reaction parameter $\Delta$ increases from -0.5 to 0.0 for $S c=0.66$ while $\left(G r^{-11 / 25} C_{f x}\right)$ decreases approximately about $24 \%$ for $S c=2.64$. Furthermore, when the chemical reaction parameter $\Delta$ increases from 0.0 to $1.0,\left(G r^{-11 / 25} C_{f x}\right)$ decreases about $20 \%$ approximately for $S c=0.66$ while $\left(G r^{-11 / 25} C_{f x}\right)$ decreases approximately $14 \%$ for $S c=2.64$ (Fig. 11b). The mass transfer rate $\left(G r^{-1 / 5} S h_{x}\right)$ increases about $64 \%$ approximately as the chemical reaction parameter $\Delta$ increases from -0.5 to 0.0 for $S c=$ 0.66 while $\left(G r^{-1 / 5} S h_{x}\right)$, increases approximately $130 \%$ for $S c=2.64$. Furthermore, when the chemical reaction parameter $\Delta$ increases from 0.0 to $1.0,\left(G r^{-1 / 5} S h_{x}\right)$ increases $92 \%$ approximately for $S c=0.66$ while $\left(G r^{-1 / 5} S h_{x}\right)$ increases approximately $122 \%$ for $S c=2.64$ (Fig. 12b).

\section{Conclusions}

A detailed numerical analysis for the problem of coupled heat and mass transfer by natural convection flow of a polar fluid through a porous medium bounded by a vertical plate in the presence of first-order chemical reaction was carried out. The plate was maintained at uniform heat and mass fluxes. The governing equations were transformed into a set of non-similar equations. This set of non-similar, coupled nonlinear, partial differential equations governing the flow, heat and mass transfer was solved numerically using an implicit finite-difference scheme in combination with quasi-linearization technique. Conclusions of the study are summarized as follows:

1. The effect of the ratio of buoyancy forces $\lambda$ caused overshoot in the velocity profiles.

2. The effects of the material parameters $\alpha$ and $\beta$ were significant on the velocity, angular velocity, temperature and concentration profile.

3. The velocity of the polar fluid decreased as the material parameter $(\alpha)$ increased.

4. A relatively higher Prandtl number (such as $P r=7.0$ for water) caused thinner momentum and thermal boundary layers while it produced thicker angular momentum and concentration boundary layers.

5. The fluid velocity decreased in the presence of a porous medium as compared to the case without a porous medium.

6. The effects of the chemical reaction parameter $\Delta$ and the Schmidt number $S c$ were found to decrease the velocity and concentration profiles.

\section{Acknowledgements}

Dr. P. M. Patil wishes to express his sincere thanks to University Grants Commission, South Western Regional Office, Bangalore, India, for the financial support under the Minor Research Project No. MRP(S)77/12-13/KAKA060/UGC-SWRO. Also, Dr. Patil dedicates this paper to Dr. Ajith Prasad, Principal and Finance Officer, Janata Shikshana Samiti, Dharwad-580 004, Karnataka State, India, on his completion of sixty one years of age.

\footnotetext{
Nomenclature

C species concentration

$C_{a}, C_{d}$ coefficients of couple stress viscosities
} 
$C_{f x} \quad$ local skin-friction coefficient

$C_{p} \quad$ specific heat at constant pressure

$C_{\infty} \quad$ species concentration far away from the wall

$D \quad$ mass diffusivity

Da Darcy number

$f_{\eta} \quad$ dimensionless velocity component

$F \quad$ dimensionless velocity component

$f \quad$ dimensionless stream function

$g \quad$ acceleration due to gravity

$G r, G r^{*}$ Grashof numbers due to temperature and concentration, respectively

$h \quad$ heat transfer coefficient

hm mass transfer coefficient

I a constant of same dimension as that of the moment of inertia of unit mass

$k_{1} \quad$ first-order chemical reaction rate

$k \quad$ thermal conductivity of fluid

$K \quad$ permeability of the porous medium $\left(\mathrm{m}^{2}\right)$

$L \quad$ characteristic length

$m_{w} \quad$ mass flux per unit area at the plate

$N u_{x} \quad$ local Nusselt number

Pr Prandtl number

$q_{w} \quad$ heat flux per unit area at the plate

Sc Schmidt number

$S h_{x} \quad$ local Sherwood number

$T \quad$ temperature in the boundary layer

$T_{\infty} \quad$ temperature of fluid far away from the wall

$u, v \quad$ components of velocities along and perpendicular to the plate, respectively

$\mathrm{x}, \mathrm{y} \quad$ coordinate system

Greek symbols

$\alpha, \beta \quad$ material parameters characterizing the polarity of the fluid

$\beta_{T}, \beta_{C}$ volumetric coefficients of the thermal and concentration expansions, respectively

$\gamma \quad$ spin gradient

$\psi \quad$ streamfunction

$\Delta \quad$ chemical reaction parameter

$\Theta, \Phi$ dimensionless temperature and concentration, respectively

$\omega \quad$ angular velocity of rotation of particles

$\lambda \quad$ buoyancy ratio

$\rho \quad$ density of the fluid

$\mu \quad$ dynamic viscosity of the fluid

$\mu_{r} \quad$ rotational dynamic viscosity

$v \quad$ kinematic viscosity

$v_{r} \quad$ rotational kinematic viscosity

$\Omega \quad$ dimensionless angular velocity

$\xi, \eta \quad$ transformed variables

\section{Subscripts}

$C$ of species concentration

$T \quad$ of temperature

w condition at wall 
$\xi, \eta \quad$ denote the partial derivatives w.r.t. these variables, respectively

\section{References}

[1] Cheng, P., Heat transfer in geothermal systems, Adv. Heat Transfer, 15 (1978), 1 - 105.

[2] Lee, S. L., Chen, T. S., Armaly, B. F., Mixed convection along vertical cylinders and needles with uniform surface heat flux, ASME J. Heat Transfer, 109 (1987), $711-716$.

[3] Chen, H.T., Chen, C. K., Natural convection of non-Newtonian fluids along a vertical plate embedded in a porous medium, ASME Journal of Heat Transfer, 110 (1988), $257-260$.

[4] Nakayama, A., Koyama, H., Buoyancy induced flow of non-Newtonian fluids over a non-isothermal body of arbitrary shape in a fluid saturated porous medium, Applied Scientific Research, 48 (1991), 55 - 70.

[5] Kim, G. B., Hyun, J. M., Buoyant convection of power-law fluid in an enclosure filled with heat generating porous media, Numerical Heat Transfer, Part A: Applications. 45 (2004), 569 - 582.

[6] Rastogi, S. K., Poulikakos, D., Double diffusion from a vertical surface in a porous region saturated with a non-Newtonian fluid, Int. J. Heat Mass Transfer, 38 (1995), 935 - 946.

[7] Jumah, R.Y., Mujumdar, A.S., Free convection heat and mass transfer of non-Newtonian power law fluids with yield stress from a vertical plate in saturated porous media, Int. Commun. Heat and Mass Transfer, 27 (2000), 485 - 494.

[8] Jumah, R.Y., Mujumdar, A.S., Free convection heat and mass transfer from a vertical plate with variable wall temperature and concentration to power law fluids with yield stress in a porous medium, Chemical Engineering Communications, 185 (2001), 165 182.

[9] Cheng, C.Y., Natural convection heat and mass transfer of non-Newtonian power law fluids with yield stress in porous media from a vertical plate with variable heat and mass fluxes, Int. Commun. Heat and Mass Transfer, 33 (2006), 1156 - 1164.

[10] Aero, E.L., Bulygin, A.N., Kuvshinski, E.V., Asymmetric hydromechanics, J. Appl. Math. Mech, 29 (1965), $333-346$.

[11] D'ep, Nguen Van, Equations of a fluid boundary layer with couple stresses, J. Appl. Math. Mech., 32 (1968), 777 - 783.

[12] Cowin, S.C., The Theory of polar fluids, Adv. Appl. Mech., 14 (1974), $279-347$.

[13] Patil, P. M., Hiremath, P. S., A note on the effect of couple stresses on the flow through a porous medium, Rheol Acta, 21 (1992), 206- 207.

[14] Hiremath, P.S., Patil, P. M., Free convection effects on the oscillatory flow of a couple stress fluid through a porous medium, Acta Mechanica, 98 (1993), 143- 158.

[15] Helmy, K. A., MHD unsteady free convection flow past a vertical porous plate, ZAMM, 78 (1998), $255-270$.

[16] Raptis, A., Takhar, H. S., Polar fluid through a porous medium, Acta Mechanica, 135 (1999), 91-93.

[17] Kim, Y. J., Unsteady MHD convection flow of polar fluids past a vertical moving porous plate in a porous medium, Int. J. Heat Mass Transfer, 44 (2001), $2791-2799$.

[18] Ogulu, A., On the oscillating plate temperature flow of a polar fluid past a vertical porous plate in the presence of couple stresses and radiation, Int. Comm. Heat Mass Transfer, 32 (2005), 1231- 1243.

[19] Patil, P. M., Kulkarni, P. S., Effects of chemical reaction on free convective flow of a polar fluid through a porous medium in the presence of internal heat generation, Int. J. Thermal Sciences, 47 (2008), $1043-1054$.

[20] Patil, P. M., Effects of free convection on the oscillatory flow of a polar fluid through a porous medium in presence of variable heat flux, Journal of Engineering Physics and Thermophysics, 81(5) (2008), 905 - 922.

[21] Patil, P. M., Kulkarni, P. S., Free convective oscillatory flow of a polar fluid through a porous medium in the presence of oscillating suction and temperature, Journal of Engineering Physics and Thermophysics, 82(6) (2009), $1138-1145$.

[22] Chang, C. L., Lee, Z. Y., Free convection on a vertical plate with uniform and constant heat flux in a thermally stratified micropolar fluid, Mechanics Research Communications, 35 (2008), 421 - 427.

[23] Skelland, A.H.P., Diffusion of Mass Transfer, Wiley, New York (1974).

[24] Levenspiel, O., Chemical Reaction Engineering, $3^{\text {rd }}$ Edition, Wiley, New York (1999).

[25] Fairbanks, D. F., Wilke, C.R., Diffusion and chemical reaction in an isothermal laminar flow along a soluble flat plate, Ind. Eng. Chem. Res., 42 (1950), $471-475$.

[26] Van Genuchten, M. Th., Analytical solution for chemical transport with simultaneous adsorption, zeroth order production and first order decay, J. Hydrology, 49 (1981), 213 - 233.

[27] Das, U. N., Deka, R. K., Soundalgekar, V.M., Effects of mass transfer on the flow past an impulsively started infinite vertical plate with constant heat flux and chemical reaction, Forschung im Ingenieurwes - Engineering Research, 60 (1994), 284 - 287.

[28] Muthucumaraswamy, R., Ganesan, P., On impulsive motion of a vertical plate with heat flux and diffusion of chemically reactive species, Forsch. Ingenieurwes, 66 (2000), 17- 23.

[29] Muthucumaraswamy, R., Ganesan, P., First order chemical reaction on flow past an impulsively started vertical plate with uniform heat and mass flux, Acta Mechanica, 147 (2001), 45- 57.

[30] Seddeek, M. A., Finite element method for the effects of chemical reaction, variable viscosity, thermophoresis and heat generation / absorption on a boundary layer hydromagnetic flow with heat and mass transfer over a heat surface, Acta Mechanica, 177 (2005), 118.

[31] Kandasamy, R., Perisamy, K., Sivagnana Prabhu, K. K., Effects of chemical reaction, heat and mass transfer along a wedge with heat source and concentration in the presence of suction or injection, Int. J. Heat Mass Transfer, 48 (2005a), 1388-1394.

[32] Kandasamy, R., Perisamy, K., Sivagnana Prabhu, K. K., Chemical reaction, heat and mass transfer on MHD flow over a vertical stretching surface with heat source and thermal stratification effects, Int. J. Heat Mass Transfer, 48\ (2005b), $4557-4561$.

[33] Raptis, A., Perdikis, C., Viscous flow over a non-linearly stretching sheet in the presence of a chemical reaction and magnetic field, Int. J. Nonlinear Mech., 42 (2006), 527- 529.

[34] Inouye, K., Tate, A., Finite difference version quasilinearisation applied to boundary layer equations. AIAA Journal, 12 (1974), 558560 .

[35] Patil, P. M., Roy, S., Unsteady mixed convection flow from a moving vertical plate in a parallel free stream: Influence of heat generation or absorption, Int. J. Heat Mass Transfer, 53 (2010), 4749 - 4756.

[36] Schlichting, H., Boundary Layer Theory, Springer, New York, 2000.

[37] Varga, R. S., Matrix Iterative Analysis, Prentice Hall, 2000. 
Table 1. Comparison of $N u_{x} G r_{x}^{-1 / 5}$ for selected values of $\operatorname{Pr}$ to previously published work with $\alpha=0, \Delta=0, \lambda=0$ and $1 / \mathrm{Da}=0$.

\begin{tabular}{|l|l|l|l|}
\hline$P r$ & Lee et al. [2] & Chang and Lee [22] & Present results \\
\hline 0.1 & 0.2634 & 0.2634 & 0.263914 \\
\hline 0.7 & 0.4838 & 0.4838 & 0.483776 \\
\hline 7.0 & 0.8697 & 0.8697 & 0.870026 \\
\hline 100.0 & 1.5546 & 1.5532 & 1.556758 \\
\hline
\end{tabular}

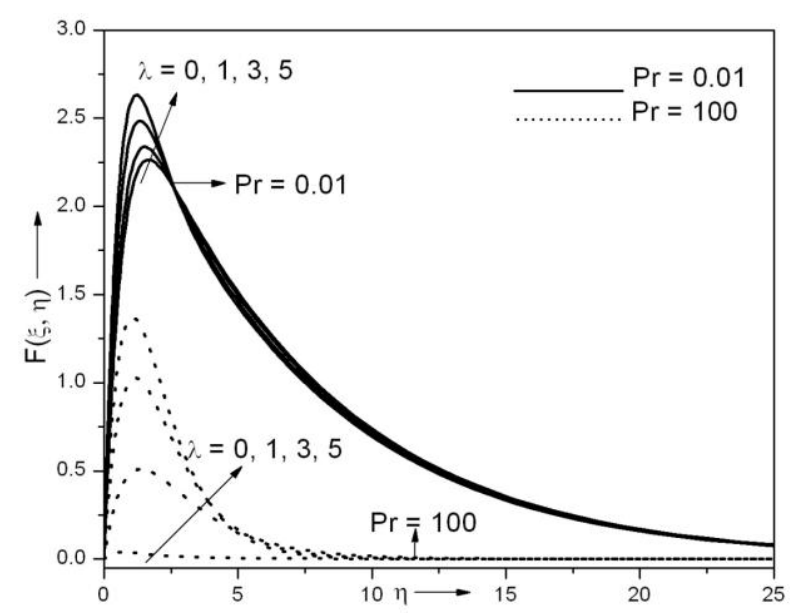

Fig. 2a: Effects of $\lambda$ and $\operatorname{Pr}$ on velocity profile for $\alpha=1$, $\beta=4, \Delta=0.5, \mathrm{Da}=1, \xi=1$ and $S c=0.22$.

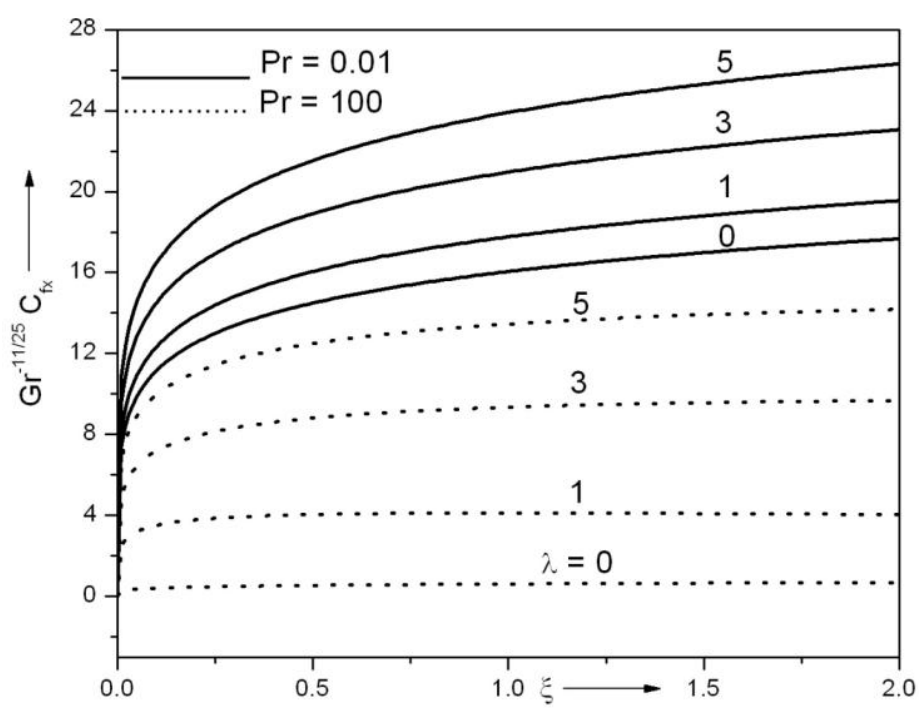

Fig. 2b: Effects of $\lambda$ and Pron skin friction coefficient for $\alpha=1, \beta=4, \Delta=0.5, D a=1$ and $S c=0.22$. 


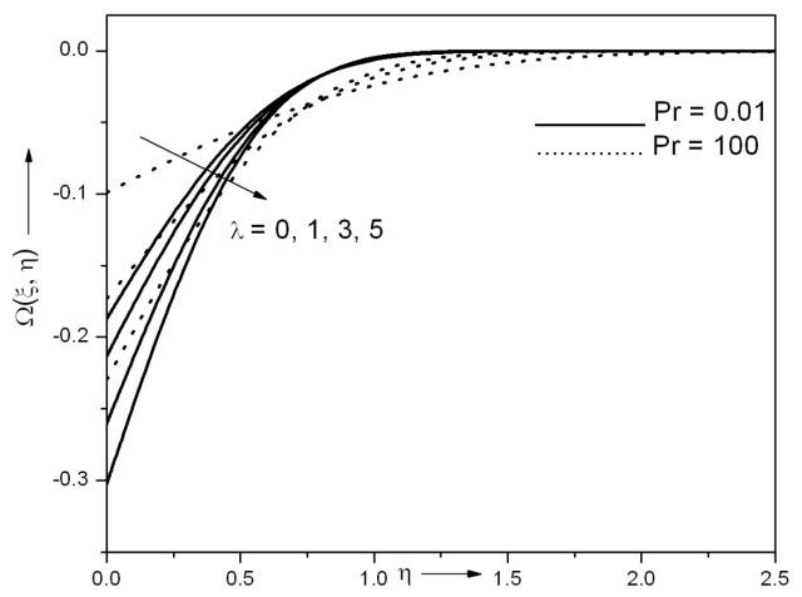

Fig. 3a: Effects of $\lambda$ and $\operatorname{Pr}$ on angular velocity profile for $\alpha=1, \beta=4, \Delta=0.5, D a=1, \xi=1$ and $S c=0.22$.

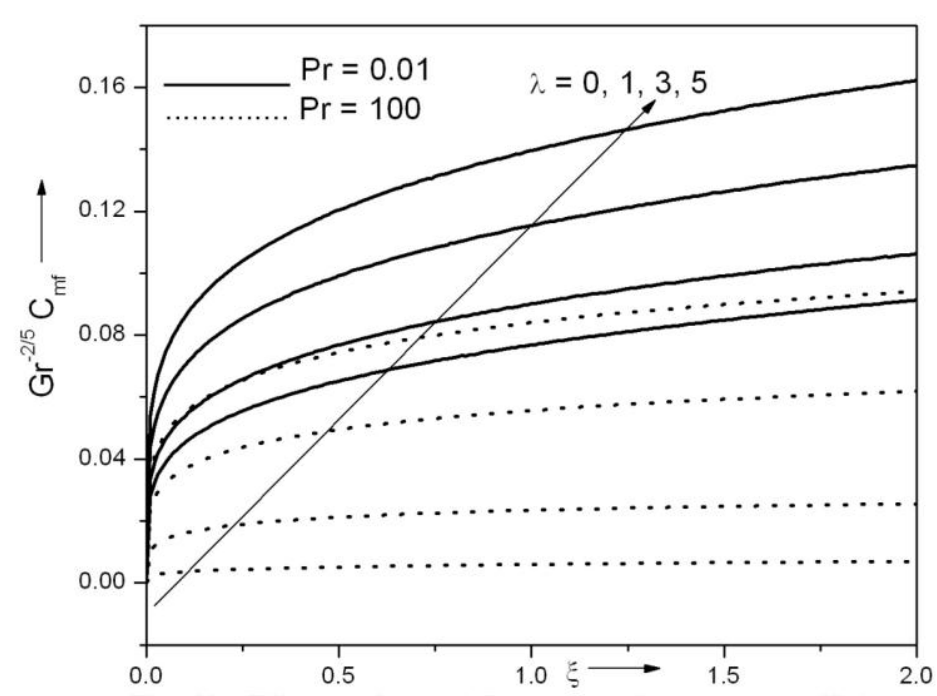

Fig. 3b: Effects of $\lambda$ and Pron couple stress coefficient for $\alpha=1, \beta=4, \Delta=0.5, D a=1, B=1$ and $S c=0.22$.

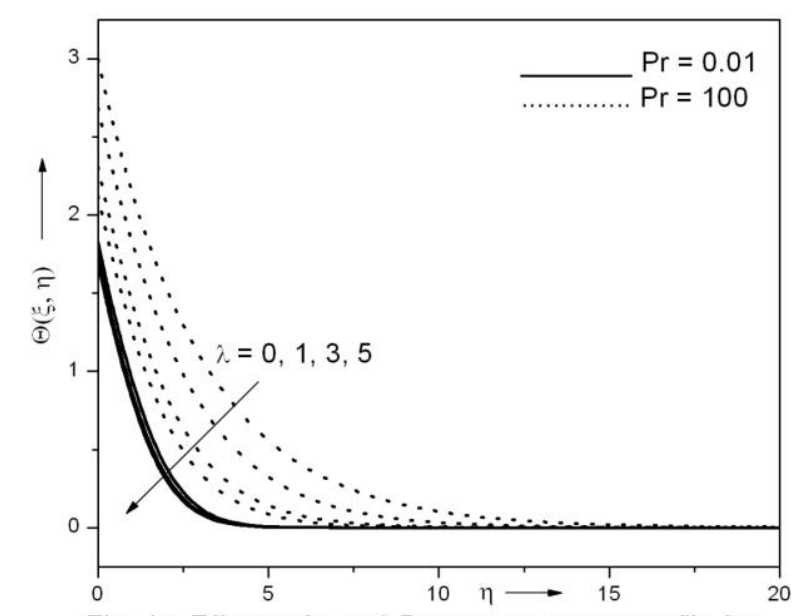

Fig. 4a: Effects of $\lambda$ and $\operatorname{Pr}$ on temperature profile for $\alpha=1, \beta=4, \Delta=0.5, D a=1, \xi=1$ and $S c=0.22$. 


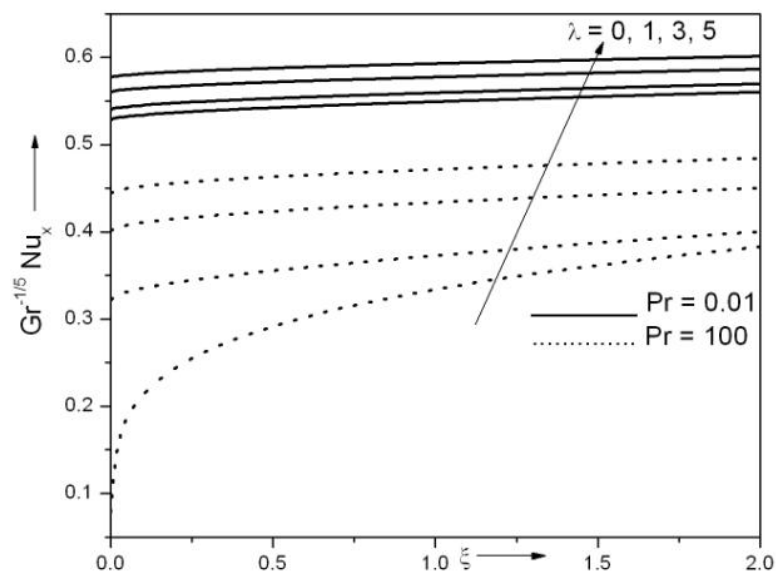

Fig. 4b: Effects of $\lambda$ and $\operatorname{Pr}$ on heat transfer rate for $\alpha=1$, $\beta=4, \Delta=0.5, D a=1, \xi=1$ and $S c=0.22$.

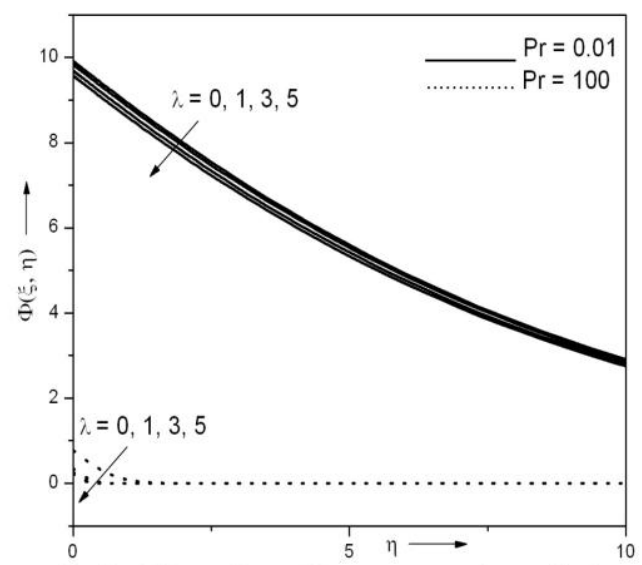

Fig. 5a: Effects of $\lambda$ and Pr on concentration profile for $\alpha=1, \beta=4, \Delta=0.5, D a=1, \xi=1$ and $S C=0.22$.

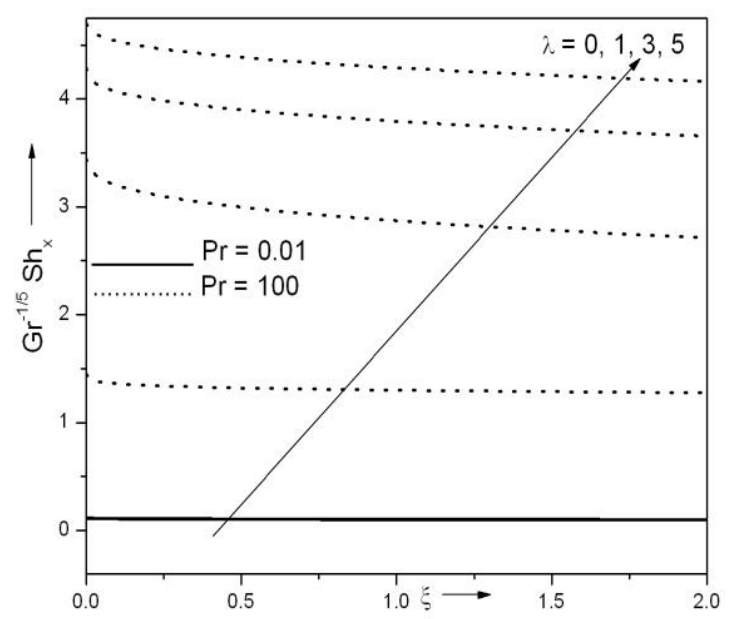

Fig. 5b: Effects of $\lambda$ and $\operatorname{Pr}$ on mass transfer rate for $\alpha=1, \beta=4, \Delta=0.5, D a=1, \xi=1$ and $S c=0.22$. 


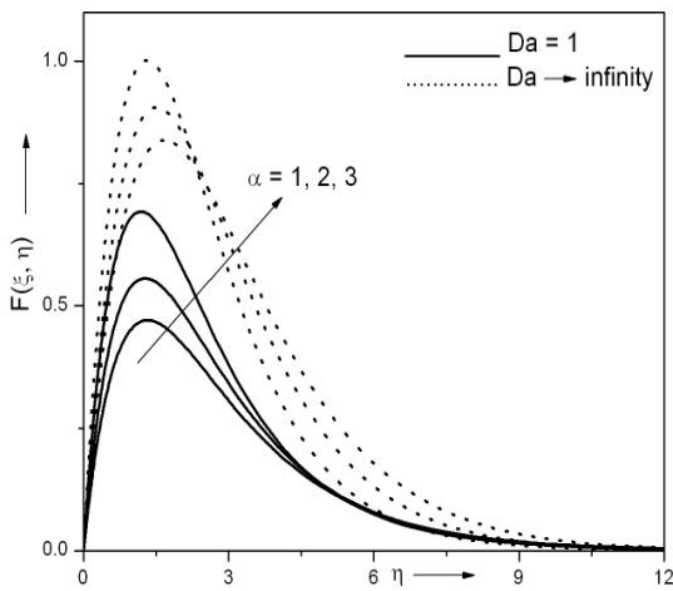

Fig. 6a: Effects of $\alpha$ and $D a$ on velocity profile for $\lambda=1$, $\beta=4, \Delta=0.5, \operatorname{Pr}=0.7, \xi=1$ and $S c=0.22$

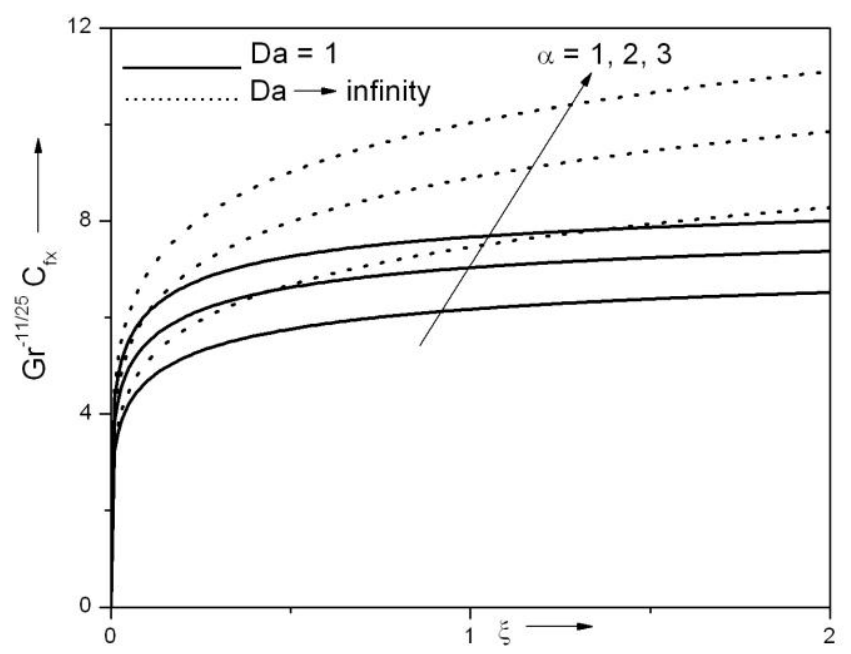

Fig. 6b: Effects of $\alpha$ and $D a$ on skin friction coefficient for $\lambda=1, \beta=4, \Delta=0.5, \operatorname{Pr}=0.7$ and $S c=0.22$.

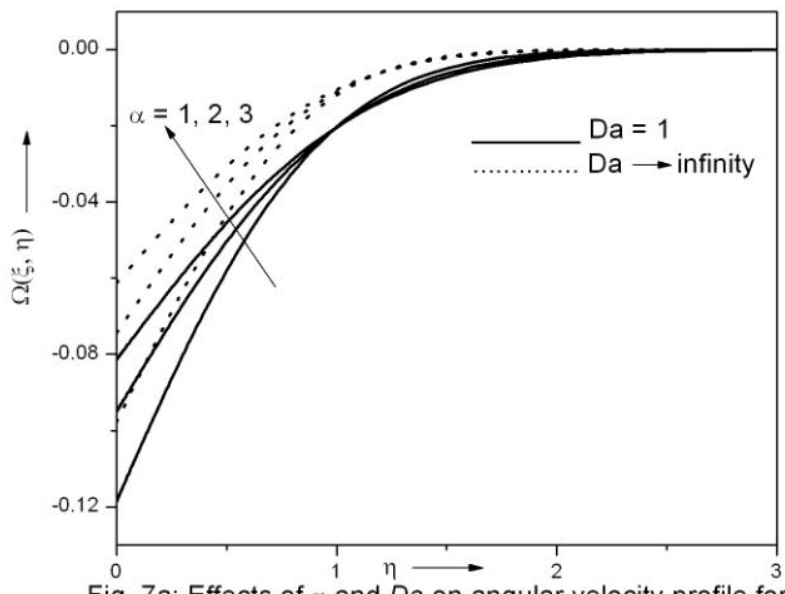

Fig. 7a: Effects of $\alpha$ and $D a$ on angular velocity profile for $\lambda=1, \beta=4, \Delta=0.5, \operatorname{Pr}=0.7, \xi=1$ and $S c=0.22$ 


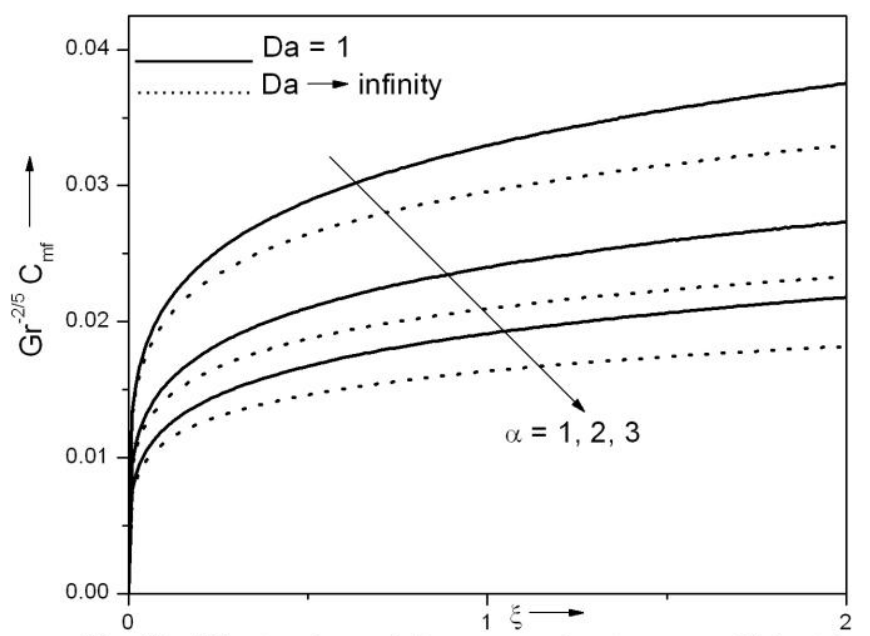

Fig. 7b: Effects of $\alpha$ and $D a$ on couple stress coefficient for $\lambda=1, \beta=4, \Delta=0.5, \operatorname{Pr}=0.7$ and $S c=0.22$.

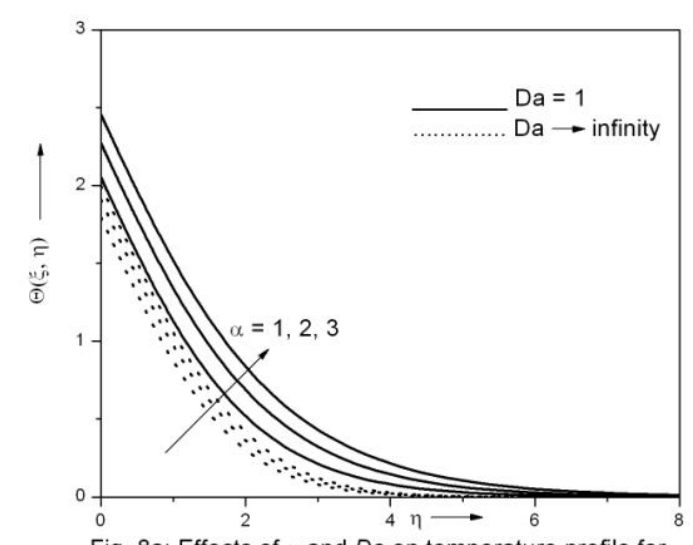

Fig. 8a: Effects of $\alpha$ and $D a$ on temperature profile for $\lambda=1, \beta=4, \Delta=0.5, \operatorname{Pr}=0.7, \xi=1$ and $S c=0.22$.

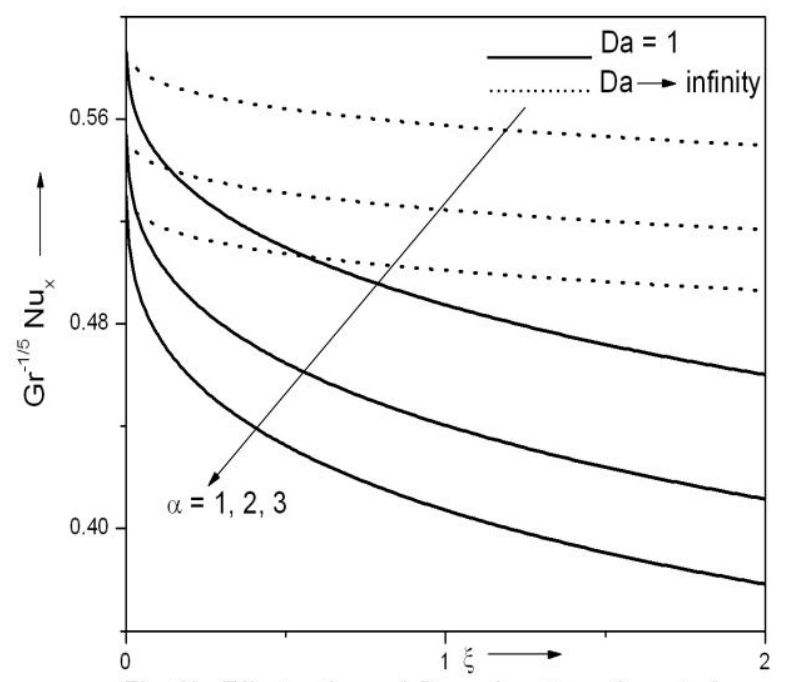

Fig. 8b: Effects of $\alpha$ and $D a$ on heat transfer rate for $\lambda=1, \beta=4, \Delta=0.5, \operatorname{Pr}=0.7$ and $S c=0.22$. 

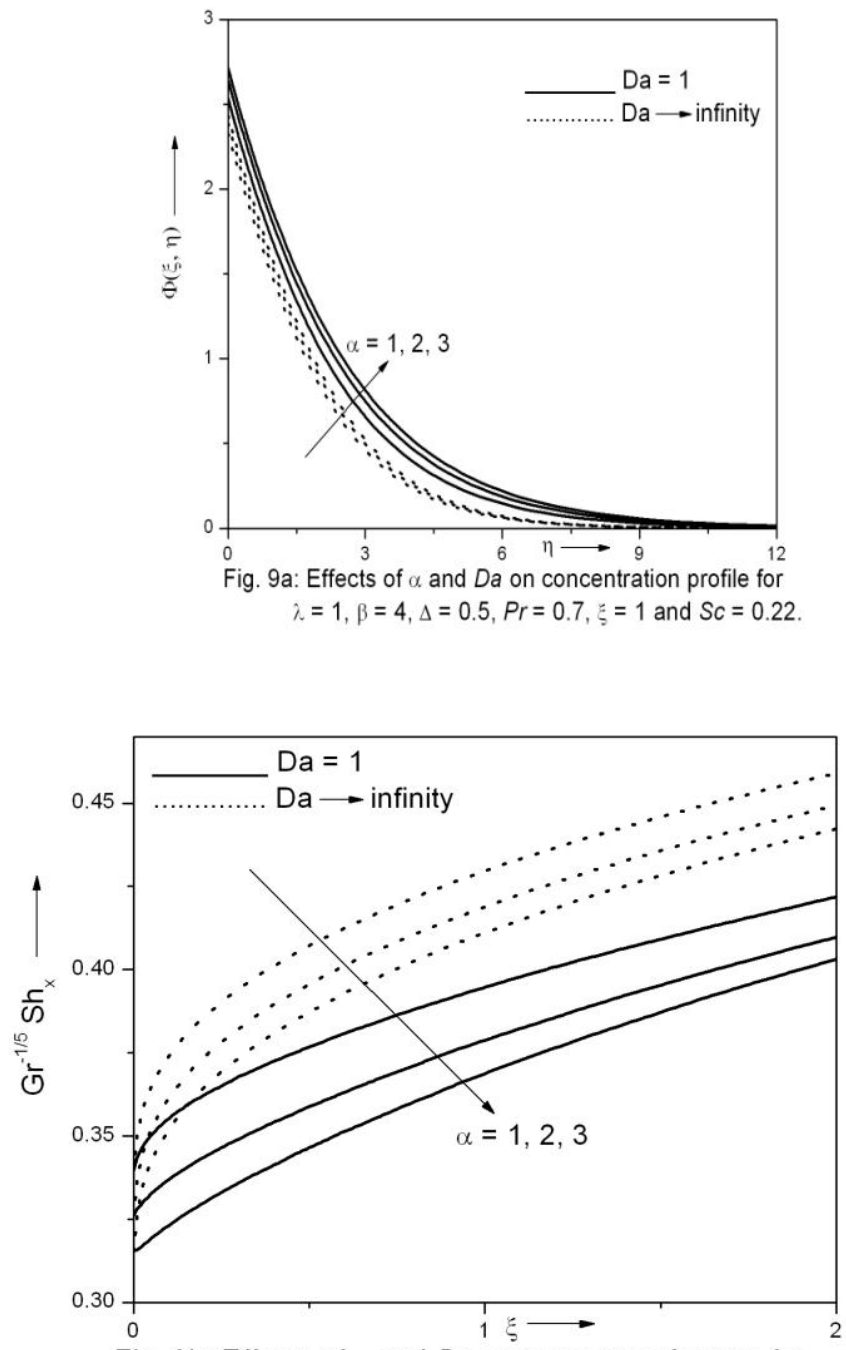

Fig. 9b: Effects of $\alpha$ and $D a$ on mass transfer rate for $\lambda=1, \beta=4, \Delta=0.5, \operatorname{Pr}=0.7$ and $S c=0.22$.

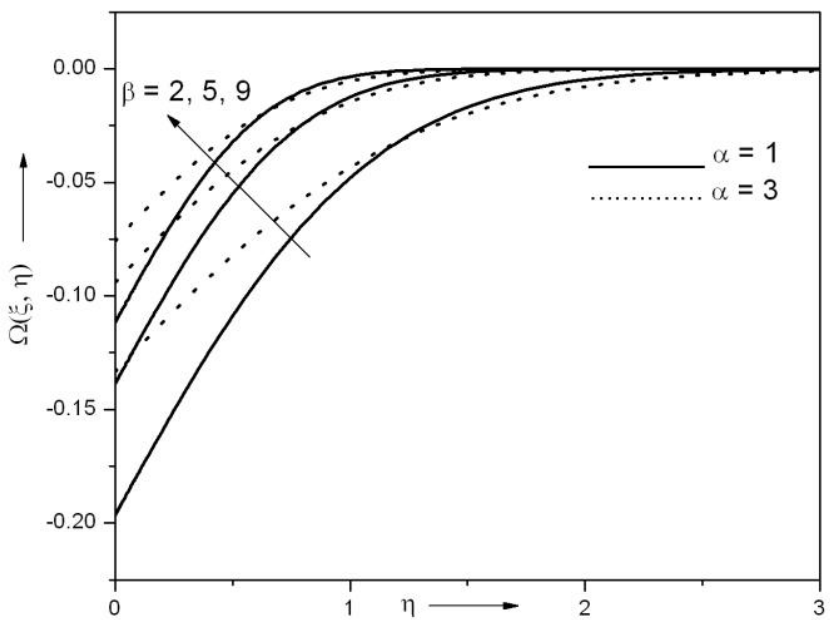

Fig. 10a: Effects of $\alpha$ and $\beta$ on angular velocity profile for $\lambda=2, D a=1, \Delta=0.5, \operatorname{Pr}=0.7, \xi=1$ and $S c=0.22$. 


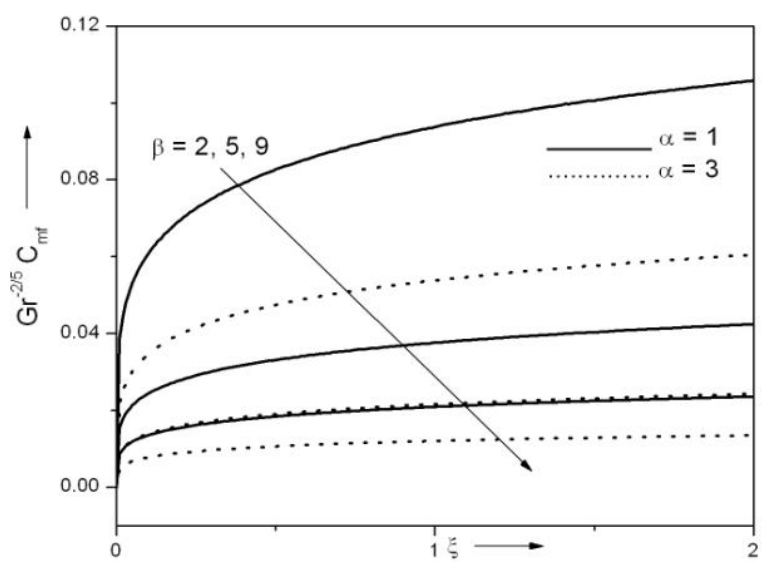

Fig. 10b: Effects of $\alpha$ and $\beta$ on couple stress coefficient for $\lambda=2, D a=1, \Delta=0.5, \operatorname{Pr}=0.7, B=1$ and $S c=0.22$.

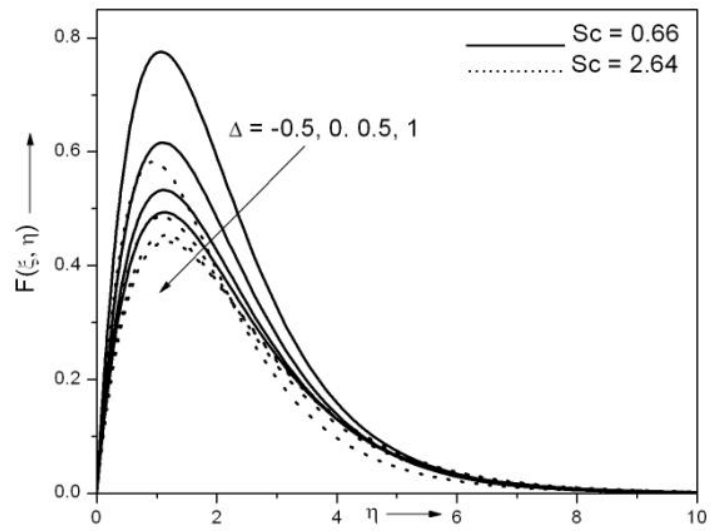

Fig. 11a: Effects of $\Delta$ and $S C$ on velocity profile for $\lambda=1$, $D a=1, \alpha=1, \beta=4, \operatorname{Pr}=0.7$ and $\xi=1$

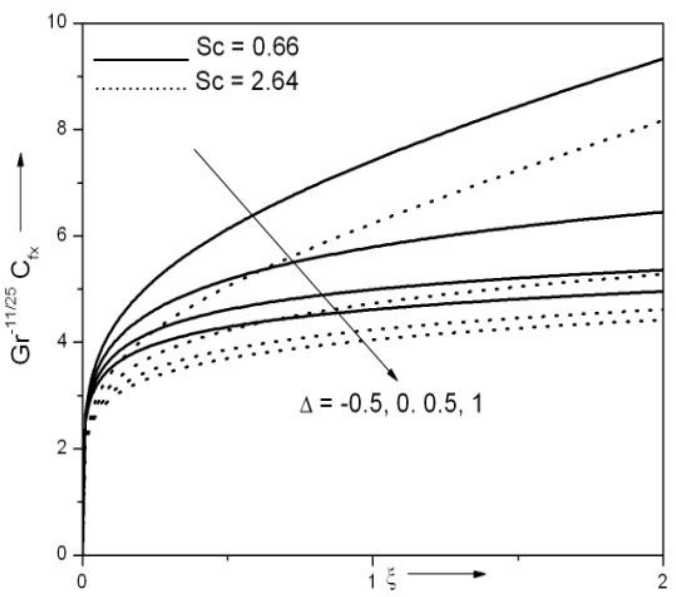

Fig. 11b: Effects of $\Delta$ and $S c$ on skin friction coefficient for $\lambda=1, \beta=4, D a=1, \operatorname{Pr}=0.7$ and $\alpha=1$. 


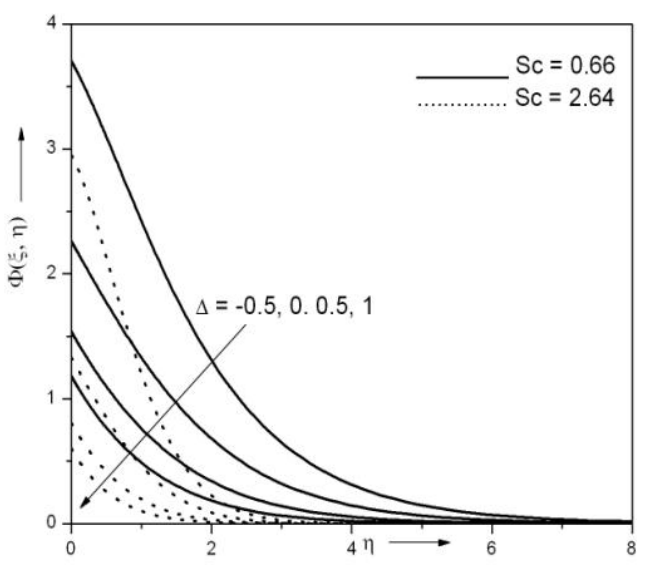

Fig. 12a: Effects of $\Delta$ and Sc on concentration profile for $\lambda=1, D a=1, \alpha=1, \beta=4, \operatorname{Pr}=0.7$ and $\xi=1$

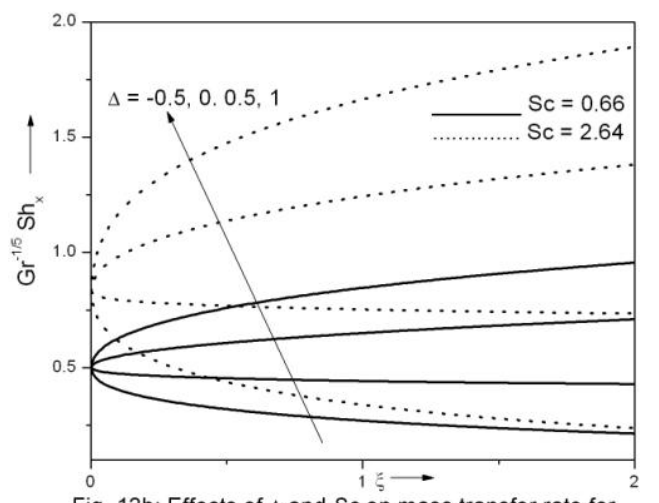

Fig. 12b: Effects of $\Delta$ and Sc on mass transfer rate for $\lambda=1, D a=1, \alpha=1, \beta=4$ and $\operatorname{Pr}=0.7$. 